\title{
Radical prostatectomy versus high intensity focused ultrasound for localized prostate cancer: a matched pair comparison
}

\author{
Albert Gelet ${ }^{1 *}$, Sebastien Crouzet ${ }^{2}$, Olivier Rouviere ${ }^{2}$, Jean-Yves Chapelon ${ }^{3}$, Murielle Rabilloud ${ }^{2}$ \\ From Current and Future Applications of Focused Ultrasound 2014. 4th International Symposium \\ Washington, D.C, USA. 12-16 October 2014
}

\section{Background/introduction}

Radical prostatectomy is the gold standard treatment for localized prostate cancer. HIFU is a treatment option with promising outcomes. No randomized study is available to compare those techniques. The goal of the study was to evaluate the oncologic outcome of patients treated with HIFU and radical prostatectomy by using a matched pair analysis to compare the 2 groups.

\section{Methods}

A total of 710 patients treated between 2000 and 2005 were prospectively followed in our institutional database and matched to a 1:1 basis following know prognostic variables: prostate-specific antigen (PSA) level, Gleason score, and clinical stage. After matching, 588 patients (294 in each group) were further analysed. The starts of salvage external beam radiotherapy (S-EBRT) or definitive palliative androgen deprivation therapy (ADT) were primary endpoints. Other endpoints were overall, cancer specific and metastasis free survival rates: The survival rates were calculated with Kaplan-Meier estimate.

\section{Results and conclusions}

The seven years S-EBRT free survival rate was significantly lower after HIFU than after RP (62\% versus $78 \%$, $\mathrm{p}=0.001)$. The palliative androgen deprivation free rate at nine years was not significantly different between HIFU and RP ( $86 \%$ versus $87 \%, \mathrm{p}=0.271$ ). At nine years the overall, cancer specific and metastasis free survival rates were similar: $89 \%, 97 \%, 94 \%$ and $89 \%, 97 \%$ and $97 \%$ for HIFU and RP respectively $(\mathrm{p}=0.186,0.312$, 0.107). Matched pair comparison of HIFU and RP has shown a higher rate of S-EBRT for HIFU. At 9 years, the rate of patients who need palliative ADT, the overall cancer specific and metastasis free survival rates were not significantly different between HIFU and RP.

\section{Acknowledgements (Funding) \\ This work was supported by the following: \\ 1. Urology and Transplantation Department, Edouard Herriot Hospital, Hospices Civils de Lyon, F-69437 Lyon, France \\ 2. Department of Genitourinary and Vascular Imaging, Edouard Herriot Hospital, Hospices Civils de Lyon, F-69437 Lyon, France \\ 3. therapeutic Applications of Ultrasound Research Laboratory, Unit 1032 (LabTau), INSERM, Lyon University, F-69003, France \\ 4. Hospices Civils de Lyon, Service de Biostatistique, Lyon, F-69003, France ; Université de Lyon, Lyon, F-69000, France}

\section{Authors' details}

'Edouard Herriot Hospital, Lyon, France. ${ }^{2}$ Hospices Civils de Lyon, Lyon, France. ${ }^{3}$ Inserm, Lyon, France.

Published: 30 June 2015

doi:10.1186/2050-5736-3-S1-056

Cite this article as: Gelet et al: Radical prostatectomy versus high intensity focused ultrasound for localized prostate cancer: a matched pair comparison. Journal of Therapeutic Ultrasound 2015 3(Suppl 1):056.

'Edouard Herriot Hospital, Lyon, France

Full list of author information is available at the end of the article 